\title{
The Cerebellum \\ The CHOPIn study: A multicenter study on Cerebellar Hemorrhage and Outcome in Preterm Infants \\ --Manuscript Draft--
}

\begin{tabular}{|c|c|}
\hline Manuscript Number: & CERE-D-19-00021R1 \\
\hline Full Title: & $\begin{array}{l}\text { The CHOPIn study: A multicenter study on Cerebellar Hemorrhage and Outcome in } \\
\text { Preterm Infants }\end{array}$ \\
\hline Article Type: & Original Article \\
\hline Corresponding Author: & $\begin{array}{l}\text { Vivian Boswinkel } \\
\text { Isala } \\
\text { NETHERLANDS }\end{array}$ \\
\hline \multicolumn{2}{|l|}{$\begin{array}{l}\text { Corresponding Author Secondary } \\
\text { Information: }\end{array}$} \\
\hline Corresponding Author's Institution: & Isala \\
\hline \multicolumn{2}{|l|}{$\begin{array}{l}\text { Corresponding Author's Secondary } \\
\text { Institution: }\end{array}$} \\
\hline First Author: & Vivian Boswinkel \\
\hline \multicolumn{2}{|l|}{ First Author Secondary Information: } \\
\hline \multirow[t]{11}{*}{ Order of Authors: } & Vivian Boswinkel \\
\hline & Sylke Steggerda \\
\hline & Monica Fumagalli \\
\hline & Alessandro Parodi \\
\hline & Luca Ramenghi \\
\hline & Floris Groenendaal \\
\hline & Jeroen Dudink \\
\hline & Manon Benders \\
\hline & Ronnie Knol \\
\hline & Linda de Vries \\
\hline & Gerda van Wezel-Meijler \\
\hline \multicolumn{2}{|c|}{ Order of Authors Secondary Information: } \\
\hline \multicolumn{2}{|l|}{ Funding Information: } \\
\hline Abstract: & $\begin{array}{l}\text { Introduction } \\
\text { Cerebellar hemorrhage }(\mathrm{CBH}) \text { is a frequent complication of preterm birth and may play } \\
\text { an important and under-recognized role in neurodevelopment outcome. Association } \\
\text { between } \mathrm{CBH} \text { size, location and neurodevelopment is still unknown. The main } \\
\text { objective of this study was to investigate neurodevelopmental outcome at two years of } \\
\text { age in a large number of infants with different patterns of } \mathrm{CBH} \text {. } \\
\text { Methods } \\
\text { Of preterm infants ( } \leq 34 \text { weeks) with known } \mathrm{CBH} \text {, perinatal factors, neuro-imaging } \\
\text { findings and follow-up at } 2 \text { years of age were retrospectively collected. MRI scans were } \\
\text { reassessed to determine the exact size, number and location of } \mathrm{CBH} \text {. CBH was } \\
\text { divided into three groups: punctate }(\leq 4 \mathrm{~mm}) \text {, limited }(>4 \text { mm but }<1 / 3 \text { of the } \\
\text { cerebellar hemisphere) or massive ( } \geq 1 / 3 \text { of the cerebellar hemisphere). Associations } \\
\text { between pattern of } \mathrm{CBH} \text {, perinatal factors and (composite) neurodevelopmental } \\
\text { outcome were assessed. }\end{array}$ \\
\hline
\end{tabular}




\section{Results}

Data of 218 preterm infants with $\mathrm{CBH}$ were analyzed. Of 177 infants the composite outcome score could be obtained. Forty-eight out of 119 infants $(40 \%)$ with punctate $\mathrm{CBH}, 18$ out of 35 infants (51\%) with limited $\mathrm{CBH}$ and 18 out of 23 infants (78\%) with massive $\mathrm{CBH}$ had an abnormal composite outcome score. No significant differences were found for the composite outcome between punctate and limited CBH $(p=0.42)$.

\section{Conclusion}

The risk of an abnormal outcome increased with increasing size of $\mathrm{CBH}$. Infants with limited $\mathrm{CBH}$ have a more favorable outcome than infants with massive $\mathrm{CBH}$. It is therefore important to distinguish between limited and massive $\mathrm{CBH}$.

\section{Response to Reviewers:}

Unfortunately we omitted to include Dr. L. Ramenghi to the list of coauthors and sincerely hope that you will still allow us to add him to the list of co-authors at this late stage. Dr. L. Ramenghi made a substantial contribution to the conception and acquisition of the CHOPIn study. He revised the final version of our manuscript critically and added important intellectual content.

All co-authors accepted and are aware of the change in the author list. 
Dear Dr Habas,

We would like to thank you for your message and the reviewer for his/her valuable comments to our manuscript The CHOPIn study: A multicenter study on Cerebellar Hemorrhage and Outcome in Preterm Infants Ms. No. CERE-D-19-00021.

We have adapted the manuscript accordingly; the changes have been marked throughout the manuscript. Below, we provide the answers to the reviewer's comments.

Additionally, we made minor adjustments to table 5 and table 6 . In table 5 the p-value for postnatal steroids was 0.00 , which is statistically impossible. This $p$-value is recalculated and has been changed to $<0.001$. Furthermore, in table 6 we added the separate $p$-values for each neurodevelopmental test using Fischer's exact test, to provide a more accurate statistical overview.

Furthermore unfortunately we omitted to include Dr. L. Ramenghi to the list of coauthors and sincerely hope that you will still allow us to add him to the list of co-authors at this late stage. Dr. L. Ramenghi made a substantial contribution to the conception and acquisition of the CHOPIn study. He revised the final version of our manuscript critically and added important intellectual content. We would also like to get permission to add S. Uccella to the acknowledgements. S. Uccella has contributed to the acquisition of follow-up data. The name of the University Hospital, Milan has been adapted, according to the newest regulations of this hospital.

We hope the manuscript has now been satisfactorily adapted and is suitable for publication in The Cerebellum.

Thank you again and yours sincerely, also on behalf of the other authors.

Vivian Boswinkel, research-physician 


\section{Answers}

Reviewer \#1: This is a retrospective study in a large multicentric cohort of very preterm infants examining the associations between 3 different patterns of cerebellar hemorrhage $(\mathrm{CBH})$ diagnosed on MRI and neurodevelopmental outcome evaluated by different tests at 2 years of age. The study also explores the associations between $\mathrm{CBH}$ and perinatal factors, $\mathrm{CBH}$ and cerebellar atrophy, and location of $\mathrm{CBH}$ and neurodevelopmental outcome. This is a well conducted study, relevant for clinical practice, especially in parental counselling. It provides useful information for future prospective studies.

We thank the reviewer for his/her positive comments.

However, I would suggest minor revisions.

Introduction

- The introduction is well structured. I would yet suggest introducing shortly the notion of cerebellar atrophy, as it is one of the factors that will be studied in association with the different patterns of $\mathrm{CBH}$.

This has now been throughout the introduction.

Patients and methods:

- The study design should appear clearly at the beginning of the section.

We have added the study design (first sentence of Patients \& Methods section).

- In the paragraph about follow-up (page 8, line 12), I am wondering why the language scale of the BSID-III is not included in the assessment. Language development is often very informative in combination with the other scales, and the reason should be explained.

Validation of the language scale of the BSID-III was not yet available during the entire study period in the Netherlands. We have explained this in the paragraph on Follow-up of the Patients and Methods section and have also added this as a limitation of the study.

We hope to report on school-age outcome of these same subjects in the future and will then include language to the evaluation.

Results:

- Page 10, line 20. It would be interesting to consider adding severe supratentorial lesions (IVH and WMI injury) as perinatal variables per pattern of $\mathrm{CBH}$. We can calculate from table 4 and 6 , how many infants per $\mathrm{CBH}$ pattern have or have not supratentorial lesions. However, as mentioned in the discussion (page 13, line 42), IVH has been associated with $\mathrm{CBH}$ in previous publications. It would be then informative to know how the different patterns of $\mathrm{CBH}$ are associated with IVH and WMI injury.

We have now added the incidences of IVH and WMI to table 1. There were no significant differences in incidences of severe IVH, WMI or VD between the three groups. This has been added to the Patients paragraph of the Results section.

Discussion:

- Page 12, line 59. I would suggest mitigating the conclusion regarding the "favorable" or "much better" prognosis of limited $\mathrm{CBH}$, also in the conclusion of the paper and abstract. Although limited $\mathrm{CBH}$ has a better prognosis compared to massive $\mathrm{CBH}$, with which I agree, this pattern still carries a risk of $51 \%$ of abnormal composite score, which may represent significant impairment for the survivors.

We agree with the reviewer that the risk of abnormal outcome of infants with limited $\mathrm{CBH}$ is still considerable. We have adapted a few sentences throughout the manuscript.

- While I understand the need for a composite outcome, could you comment on the weight of each element in the composite score? For instance, would an abnormal CBCL score weight the same as having a CP? 
We have now added this as a limitation of the study.

- Limitations: Regarding the MRI protocol which differs between centers, can you comment on the slice thickness ( $\max 3 \mathrm{~mm}$ ) allowing detection of small punctate lesions especially without SWI sequence? How did the protocol evolve over the study period?

In the discussion a paragraph is dedicated to the (different) MRI protocols and the availability of the SWI. In addition, in the Neuro-imaging paragraph of the Patients and Methods section we have now added a sentence on the availability of the SWI sequence. 
The CHOPIn study: A multicenter study on Cerebellar Hemorrhage and Outcome in Preterm Infants

V. Boswinkel ${ }^{1}$, S.J. Steggerda ${ }^{2}$, M. Fumagalli ${ }^{3,4}$, A. Parodi ${ }^{5}$, L.A. Ramenghi ${ }^{5}$, F. Groenendaal ${ }^{6}$, J. Dudink $^{6,7}$,

M.N. Benders ${ }^{6}$, R. Knol ${ }^{7}$, L.S. de $\operatorname{Vries}^{6}$, G. van Wezel-Meijler ${ }^{1}$

${ }^{1}$ Department of Neonatology, Isala Women and Children's hospital, Zwolle, the Netherlands.

${ }^{2}$ Department of Pediatrics, Division of Neonatology, Leiden University Medical Center, Leiden, the Netherlands.

${ }^{3}$ Fondazione IRCCS Ca' Granda Ospedale Maggiore Policlinico, Neonatal Intensive Care Unit, Milan, Italy.

${ }^{4}$ University of Milan, Department of Clinical Sciences and Community Health, Milan, Italy.

${ }^{5}$ Neonatal Intensive Care Unit, Istituto Giannina Gaslini, Genova, Italy.

${ }^{6}$ Department of Neonatology, University Medical Center Utrecht, Utrecht, the Netherlands.

${ }^{7}$ Department of Neonatology, Erasmus University Medical Center, Rotterdam, the Netherlands.

Keywords: Cerebellar hemorrhage, preterm infant, Magnetic Resonance Imaging, risk factors, outcome assessment.

Financial Disclosure: None of the authors have financial relationship relevant to this article.

Conflict of interest: None of the authors have conflict of interest relevant to this article. 
Corresponding author:

V. Boswinkel, MD

Isala Women and Children's hospital

Department of Neonatology (V.4.4.)

PO Box 10400,

8000 GK Zwolle, the Netherlands

Tel: +31384245055

E-mail: v.boswinkel@isala.nl 


\begin{abstract}
Introduction Cerebellar hemorrhage $(\mathrm{CBH})$ is a frequent complication of preterm birth and may play an important and under-recognized role in neurodevelopment outcome. Association between CBH size, location and neurodevelopment is still unknown. The main objective of this study was to investigate neurodevelopmental outcome at two years of age in a large number of infants with different patterns of CBH.
\end{abstract}

Methods Of preterm infants $(\leq 34$ weeks) with known $\mathrm{CBH}$, perinatal factors, neuro-imaging findings and follow-up at 2 years of age were retrospectively collected. MRI scans were reassessed to determine the exact size, number and location of $\mathrm{CBH}$. $\mathrm{CBH}$ was divided into three groups: punctate $(\leq 4 \mathrm{~mm})$, limited $(>4 \mathrm{~mm}$ but $<1 / 3$ of the cerebellar hemisphere) or massive ( $\geq 1 / 3$ of the cerebellar hemisphere). Associations between pattern of $\mathrm{CBH}$, perinatal factors and (composite) neurodevelopmental outcome were assessed.

Results: Data of 218 preterm infants with $\mathrm{CBH}$ were analyzed. Of 177 infants the composite outcome score could be obtained. Forty-eight out of 119 infants (40\%) with punctate $\mathrm{CBH}, 18$ out of 35 infants (51\%) with limited $\mathrm{CBH}$ and 18 out of 23 infants (78\%) with massive $\mathrm{CBH}$ had an abnormal composite outcome score. No significant differences were found for the composite outcome between punctate and limited CBH $(\mathrm{p}=0.42)$. Conclusion The risk of an abnormal outcome increased with increasing size of CBH. Infants with limited CBH have a more favorable outcome than infants with massive $\mathrm{CBH}$. It is therefore important to distinguish between limited and massive $\mathrm{CBH}$. 


\section{Introduction}

Cerebellar hemorrhage $(\mathrm{CBH})$ is a common complication in very low birthweight infants. Reported incidences in infants born below 32 weeks gestation, and/or weighing less than 1500 grams at birth, range from 2.2 to $19 \%$ (13), depending on the population studied and the imaging techniques used. The etiology of $\mathrm{CBH}$ is multifactorial. Potential risk factors are traumatic delivery and circulatory events related to prematurity, such as impaired cerebrovascular autoregulation, large patent ductus arteriosus, and other parameters of a compromised cerebral circulation (4-7).

Until recently it was thought that the cerebellum was mainly involved in motor system functions (8). However, in a retrospective, case-control design study, Limperopoulos et al. found cerebellar hemorrhagic injury in preterm infants to be associated with a high prevalence of long-term pervasive neurodevelopmental disabilities (2). Other studies have shown that injury to the cerebellum not only affects motor functions, but also non-motor functions, including cognition, learning, and behavioral abilities $(9,10)$. CBH may thus play an important and under-recognized role in the cognitive, learning, and behavioral problems known to affect survivors of extremely preterm birth $(6,11,12)$.

In preterm infants with $\mathrm{CBH}$ the size of the lesion may be of importance with respect to neurodevelopmental outcome. Three patterns of preterm CBH have been described $(13,14)$; the first being massive $\mathrm{CBH}$, mainly seen in the sickest and smallest infants ( $<28$ weeks gestation and/or $<750$ grams). These massive bleeds carry a high morbidity and mortality and are easily diagnosed with cranial ultrasonography (CUS), especially when the mastoid window is used $(4,15)$. In surviving infants, massive CBH leads to severe cerebellar destruction and subsequent atrophy, and is associated with long term and severe neurodevelopmental disabilities, such as cerebral palsy $(\mathrm{CP})$ and motor, language and/or cognitive delays $(1,2,11)$. Associated supratentorial injury and/or cerebellar diaschisis may also play a role in this unfavorable outcome $(5,6)$. Another pattern is small or punctate hemorrhages. These are small $(\leq 4 \mathrm{~mm})$ lesions that are beyond the scope of CUS but are frequently encountered as a chance finding on magnetic resonance imaging (MRI), which is often performed in very preterm infants around term equivalent age (TEA) (14). These small CBH do not seem to lead to cerebellar atrophy and are associated with a more favorable prognosis. Tam et al. (16) found preterm infants with small CBH to have a 5fold higher incidence of abnormal neurological examination at $3-6$ years of age than those without $\mathrm{CBH}$, but there was no significant difference in cognitive impairment. Steggerda et al. found no association between small 
$\mathrm{CBH}$ and neurodevelopmental outcome at 2 years of age (17). The third, so far only rarely described pattern concerns "medium-sized" or limited hemorrhages that involve less than one third of the cerebellar hemisphere. These limited hemorrhages are mostly located at the convexity of one of the cerebellar hemispheres and may be diagnosed with CUS, especially if the mastoid fontanel is used as an additional acoustic window (13). They occur in very preterm neonates, do not seem to cause acute clinical symptoms and their possible influence on outcome is not yet known, in addition, it is not known whether these limited hemorrhages may lead to cerebellar atrophy $(14,18)$. As different parts of the cerebellum seem to be involved in different executive, affective, limbic and sensorimotor functions, the relationship between location of the lesion(s) and neurodevelopmental and behavioral outcome is also worth investigating $(11,19-22)$.

Despite the increasing number of papers reporting about neonatal $\mathrm{CBH}$, the number of included infants with $\mathrm{CBH}$ in these studies is limited. Therefore, drawing conclusions from associations between $\mathrm{CBH}$ size and location, and neurodevelopment is still a challenge. The main objective of this study was therefore to investigate and compare neurodevelopmental outcome at two years of age in a large number of infants with punctate, limited and massive $\mathrm{CBH}$. Other objectives were to investigate associations between:

- location of $\mathrm{CBH}$ and neurodevelopmental outcome;

- $\quad$ pattern of $\mathrm{CBH}$ and cerebellar atrophy;

- $\quad$ pattern of $\mathrm{CBH}$ and perinatal factors.

\section{Patients and methods}

In this retrospective, multi-center study, data from preterm infants $<34$ weeks gestation with MRI diagnosed CBH who were admitted to one of 6 tertiary neonatal centers (4 Dutch and 2 Italian) were collected and analyzed. The participating centers were selected based on their neonatal neuro-imaging protocols with special attention for cerebellar injury. Infants were born between 2003 and 2016 and included if at least one neonatal MRI examination had been performed. Exclusion criteria were: (suspected) brain malformation, dysmorphic features or congenital anomaly suggestive of a genetic syndrome, metabolic disorder, chromosomal abnormality and/or proven central nervous system infection.

The participating centers were Isala Women and Children's hospital (IVKC), Zwolle, the Netherlands; Leiden University Medical Center (LUMC), Leiden, the Netherlands; University Medical Center Utrecht (UMCU), Utrecht, the Netherlands; Erasmus Medical Center (Erasmus MC), Rotterdam, the Netherlands; Fondazione 
IRCCS Ca’ Granda Ospedale Maggiore Policlinico, Milan, Italy; and Istituto Giannina Gaslini, Genova, Italy. Infants were selected from an institutional MRI register and from existing databases of infants known to have cerebellar abnormalities.

As the study did not fall under the Medical Research Involving Human Subjects Act and clinically obtained anonymized data were used, the medical ethical committees of the participating centers waived an informed consent and ethical review procedure.

\section{Patient characteristics}

Maternal, perinatal and neonatal factors were retrieved from the medical records. Maternal factors included age, presence of pre-eclampsia and use of antenatal steroids. Perinatal factors included gender, gestational age (GA), birth weight (BW), Z-score for BW according to Hoftiezer et al. (23), multiple birth, mode of delivery (i.e. breech extraction, instrumental delivery, caesarean section), umbilical cord pH and Apgar score at 5 minutes. Neonatal factors were mechanical ventilation within the first postnatal week, high frequency oscillation (HFO) ventilation within the first postnatal week, surfactant replacement therapy, hypotension (defined as need for inotropic support) within the first postnatal week, severe thrombocytopenia (defined as platelet count $<50 \mathrm{x}$ $10^{\wedge} 9 / \mathrm{L}$ ) within the first postnatal week, and in-hospital mortality.

\section{Neuro-imaging}

At IVKC, LUMC, UMCU, Fondazione IRCCS Ca' Granda and Gaslini, MRI was performed around term equivalent age (TEA) using a 1.5-T or 3.0-T MR system (Ingenia (IVKC), Achieva (LUMC, UMCU, Fondazione IRCCS Ca' Granda and Gaslini), Philips Medical Systems, Best, The Netherlands. At Erasmus MC MRI was obtained around the postmenstrual age (PMA) of 30 weeks, or, in infants born at GA $>30$ weeks, as soon as possible after birth, using a 1.5-T GE EchoSpeed scanner (General Electrics Healthcare Technologies, Waukesha, WI). In UMCU MRI was routinely done in all infants $<28$ weeks gestation and in Fondazione IRCCS Ca' Granda in all infants $<32$ weeks gestation, while in the other hospitals MRI was performed if there was an indication, according to the local guidelines. T1-weighted, T2-weighted images (slice thickness $1.2 \mathrm{~mm}$ at Erasmus MC; 2 mm at IVKC, LUMC, UMCU and Fondazione IRCCS Ca’ Granda; 3 mm at Gaslini) and, if available, susceptibility weighted images (SWI) were used for detection and scoring CBH. The SWI sequence was not available during the first years of the study and was only used since 2006 in some centers and since 2014 in all. Due to slice thickness of $3 \mathrm{~mm}$ for the T2-weighted images in Gaslini and therefore the limited ability to 
detect punctate $\mathrm{CBH}$, especially before the SWI-era, only infants with limited or massive $\mathrm{CBH}$ were selected from this center. All available MRI examinations were screened for the presence of CBH by one of the investigators (V.B.). Subsequently, all MRI examinations with $\mathrm{CBH}$ were reviewed by 2 investigators: V.B. along with a neonatologist of each center (G.M.; S.J.S.; L.S.V.; J.D.; A.P. or M.F.). These neonatologists are experts with at least 10 years of experience in neonatal neuro-imaging. In case of disagreement an expert from one of the other centers was consulted.

Pattern of CBH: The classification was modified from the CUS classification by Meijler and Steggerda (18) and previous work by others $(13,24)$ (Figure 1).

1. Punctate CBH: one or more lesions $\leq 4 \mathrm{~mm}$;

Infants with punctate $\mathrm{CBH}$ were subdivided into two groups: $\leq 6$ lesions or $>6$ lesions.

2. Limited CBH: the lesion(s) being $>4 \mathrm{~mm}$, but involving $<1 / 3$ of the cerebellar hemisphere;

3. Massive $\mathrm{CBH}$ : the lesion involving $\geq 1 / 3$ of the cerebellar hemisphere.

The pattern of CBH was determined based on the T2-W images (transverse planes). Foci of signal loss on T2-W images and/or SWI were considered to be hemosiderin deposits and thus (punctate) hemorrhages if there was no continuity with a vessel, suggesting a vascular structure.

For the MRI scans performed around TEA (PMA 38 - 44 weeks), the transcerebellar diameter (TCD) was measured on a transverse T2-W plane, at the largest cerebellar diameter. Because the TCD increases with increasing PMA at MRI, we corrected the measured value according to the equation developed by Kidokoro et al (25). Cerebellar atrophy was considered mild to moderate if the corrected TCD was $<50 \mathrm{~mm}$, but $\geq 44 \mathrm{~mm}$ and severe if the corrected TCD was $<44 \mathrm{~mm}$.

Location of CBH: We also categorized CBH according to location. Figure 2 shows a map of structuralfunction corresponding regions of the cerebellum on transverse and coronal planes. Region A. comprises the convexity (lateral-posterior-inferior) hemispheric zones; region B. the anterior/medial hemispheric zones; and region C. the vermis. This map is based on previous work $(19-21,26)$.

Supratentorial injury: Intraventricular hemorrhage (IVH) (classified according to Volpe (27)) was recorded based on neonatal ultrasound reports. White matter injury was graded according to Kidokoro (3): Grade 1: punctate lesions $\leq 3 \mathrm{~mm}$ in the periventricular white matter on one or both sides; Grade 2: punctate lesions present within the corticospinal tract on both sides, or $\geq 3$ lesions per hemisphere; Grade 3: extensive lesions with high signal on T1-weighted images along the wall of the lateral ventricles; Grade 4: cystic lesions in the 
periventricular white matter (this could be either cystic degeneration of periventricular hemorrhagic infarction or

cystic periventricular leukomalacia).Ventricular dilatation (VD) was assessed on axial T2-weighted images at midventricular level. Bilateral $\geq 7,5 \mathrm{~mm}$ or one-sided $\geq 10 \mathrm{~mm}$ VD were considered severe. Based on these criteria, infants were categorized into presence or absence of severe supratentorial injury (i.e. IVH grade 3 and 4 , WMI grade 3 and 4, and/or severe VD).

\section{Follow-up}

Results of the neurodevelopmental tests and neurological examinations performed between 18 to 36 months were retrieved from the medical files. In the four Dutch centers a standard neurological examination and the Bayley scales of infant development (BSID-III American or Dutch edition) (28) were performed. As validation of the language scale of the BSID-III was not yet available during the entire study period in the Netherlands, only the cognitive and motor scales were tested. In addition, the Child Behavior Checklist (CBCL) - a questionnaire filled out by the parents - was used as a parameter of neurodevelopment (29). Italian infants were examined and tested by means of a standard neurological examination and the Griffiths Mental Development Scales Revised (GMDS) (30). The GMDS test comprises 5 subscales: Locomotor, Personal-Social, Hearing and Speech, Eye and Hand Coordination and Performance. The results of the neurological examination were considered abnormal when infants had cerebral palsy (hemi-, di- or tetraplegia) and/or neurosensory hearing loss. Cerebral palsy was scored according to the Gross Motor Function Classification System (GMFCS) (31), a score $\geq 1$ was considered abnormal. Information on cerebral visual impairment could not be consistently retrieved from the medical records and was therefore not included. A score of $<-1$ SD for one of the subscales of the BSID-III or GMDS was considered abnormal, while a CBCL score above 60 was considered to be in the clinical range $(29,32,33)$ and thus abnormal. If at least one of the outcome parameters (neurological examination, BSIDIII or GMDS, CBCL) was abnormal, this was considered an abnormal composite outcome. Firstly, each outcome parameter was compared between infants with punctate, limited and massive CBH. Secondly, the composite outcome was compared between infants with punctate, limited and massive CBH. 


\section{Statistical analysis}

Statistical analysis was performed using SPSS software (version 23.0, SPSS Inc, Chicago, IL, USA).

Distribution of continuous variables was objectively assessed by means of the Shapiro-Wilk test. Continuous data are presented as median (range). Categorical variables are presented using frequency counts and percentages. Differences between variables were tested for significance using the Mann-Whitney-U test when comparing two continuous variables, and the Kruskal-Wallis test when comparing three variables. For categorical variables the Chi-square test or Fisher's exact test was used. The contribution of maternal, perinatal and neonatal variables on the pattern of $\mathrm{CBH}$ was analyzed using logistic regression. Neurodevelopmental outcome was related to size of $\mathrm{CBH}$ using logistic regression, adjustments were made for GA, gender and presence of severe supratentorial injury. Significance and odds ratio (OR) with $95 \%$ confidence interval (CI) were calculated and a P-value $<0.05$ was considered significant. Infants with missing data were not included in analyses. 


\section{Results}

\section{Patients}

Data of 218 infants with CBH were collected. Median GA at birth was 27.2 weeks $(23.0-34.0$ weeks $)$ and median BW 958 grams (400 - 2665 grams). Median PMA at MRI was 41.3 weeks (27.7 - 64.1 weeks). One hundred forty-seven (67\%) infants had punctate, 40 (18\%) limited and 31 (14\%) massive CBH. Severe supratentorial injury was seen in $52 \%$ of the infants with punctate, $60 \%$ of the infants with limited and $52 \%$ of the infants with massive CBH. There were no significant differences in incidences of severe IVH, WMI or VD between the three groups (see table 1). Ten infants died: two due to massive $\mathrm{CBH}$, three due to severe supratentorial injury. The five other infants died due to systemic complications (i.e. sepsis, multi-organ failure, respiratory complications).

\section{Perinatal factors and pattern of $\mathrm{CBH}$}

Table 1 shows perinatal variables for infants with punctate, limited and massive CBH. Due to missing data pvalues for mode of delivery and umbilical cord $\mathrm{pH}$ were not representative and are therefore not shown. Infants with limited and massive $\mathrm{CBH}$ had a lower GA than infants with punctate $\mathrm{CBH}$. They also experienced more respiratory difficulties: more infants with limited and massive $\mathrm{CBH}$ needed mechanical ventilation and surfactant replacement therapy. Lower 5 min Apgar score was more often seen in infants with massive $\mathrm{CBH}$ than in the other two groups. Infants with massive CBH more often needed high frequency ventilation, had lower BW, and more often severe thrombocytopenia than infants with punctate $\mathrm{CBH}$. Analyzing the contribution of these variables by logistic regression revealed GA, Apgar score and severe thrombocytopenia as independent contributors to massive $\mathrm{CBH}$, while mechanical ventilation remained an independent factor for limited $\mathrm{CBH}$.

\section{Cerebellar atrophy}

Severe atrophy $(\mathrm{TCD}<44 \mathrm{~mm})$ at MRI around TEA $(\mathrm{N}=141)$ was seen in $1 \%(1 / 102)$ of infants with punctate $\mathrm{CBH}, 26 \%(6 / 23)$ of infants with limited $\mathrm{CBH}$ and in $75 \%(12 / 16)$ of infants with massive $\mathrm{CBH}$. Mild to moderate cerebellar atrophy $(\mathrm{TCD}<50 \mathrm{~mm}$ and $\geq 44 \mathrm{~mm}$ ) was seen in $28 \%, 48 \%$ and in $19 \%$ of infants with respectively punctate, limited and massive $\mathrm{CBH}$. TCD was lower with increasingly larger $\mathrm{CBH}(\mathrm{p}<0.01$; see table 2). 


\section{Pattern of CBH and neurodevelopmental outcome}

Follow up was available for 177/208 (85\%) of surviving infants (table 3). The median corrected age at follow-up was 24.0 months (range: 18.0 - 36.0 months). Infants with massive $\mathrm{CBH}$ had the highest percentage of abnormal results for neurological examination, BSID-III, GMDS and CBCL (see table 4). For all outcome parameters, except the BSID-III, there was a trend towards higher percentages of abnormal results with an increase in the size of $\mathrm{CBH}$. As there was a striking difference between the test results of the GMSD and the Bayley-III, we analyzed the difference between Italian and Dutch children. Italian infants were significantly younger and had more respiratory difficulties, more often culture proven late onset sepsis and more surgery before TEA than Dutch infants (see table 5).

A sub-analysis, performed in infants without severe supratentorial injury $(\mathrm{N}=87)$, showed a trend towards higher percentages of abnormal results with increasingly larger $\mathrm{CBH}$ for an abnormal neurological examination and $\mathrm{CBCL}$ (table 6). Overall, 33\% of infants without severe supratentorial injury had an abnormal composite outcome. The composite outcome differed significantly between the three groups $(p<0.01)$ and again the percentages of infants with abnormal outcome increased with larger hemorrhages. Differences between the three groups remained significant ( $\mathrm{p}$ 0.02) in infants without severe supratentorial injury.

Comparing massive and punctate $\mathrm{CBH}$, logistic regression analysis demonstrated an increased risk of an abnormal composite outcome in infants with massive $\mathrm{CBH}$, with an adjusted OR of 5.52 (95\%CI $1.75-17.43$; p $<0.01)$. Comparing massive with limited $\mathrm{CBH}$, the adjusted OR was $4.09(95 \% \mathrm{CI} 1.09-15.28 ; \mathrm{p}=0.04)$. No significant differences were found for the composite outcome between punctate and limited $\mathrm{CBH}(\mathrm{p}=0.42)$.

In a sub-analysis of infants with punctate $\mathrm{CBH}$, we analyzed the neurodevelopmental outcome of infants with $\leq$ 6 punctate lesions as compared to infants with $>6$ punctate lesions. Thirty-nine infants $(40 \%)$ with $\leq 6$ punctate lesions had an abnormal composite outcome score compared to eight infants (36\%) of infants with more than six punctate lesions $(\mathrm{p}=0.79)$.

\section{Location of CBH and neurodevelopmental outcome}

Location A and location B were most frequently seen in infants with punctate and limited $\mathrm{CBH}$. A combination of location $\mathrm{A}$ and $\mathrm{B}$, and of location $\mathrm{A}, \mathrm{B}$ and $\mathrm{C}$ was most often seen in infants with massive CBH. Most infants 
$(\mathrm{N}=119)$ had unilateral $\mathrm{CBH}$. Although not significant, $67 \%$ of infants with bilateral limited $\mathrm{CBH}$ and $100 \%$ of infants with bilateral massive $\mathrm{CBH}$ had an abnormal composite outcome compared to respectively $48 \%$ and $74 \%$ of infants with unilateral limited and massive $\mathrm{CBH}$ (see table 7). In none of the infants with limited $\mathrm{CBH}$, location $\mathrm{C}$ (the vermis) was involved. For infants with punctate $\mathrm{CBH}$, outcome did not differ between infants with and without vermis involvement. All infants with massive $\mathrm{CBH}$ and vermis involvement had an abnormal composite outcome (see table 8). Due to the restricted number of infants per specific location, the relation between location and neurodevelopmental outcome could not be further investigated.

\section{Discussion}

We analyzed associations between perinatal factors, cerebellar atrophy, neurodevelopmental outcome and pattern of $\mathrm{CBH}$ determined according to a newly defined MRI classification of $\mathrm{CBH}$ that includes punctate, limited and massive $\mathrm{CBH}$. Several studies have demonstrated an increased risk of an impaired neurodevelopmental outcome for infants with $\mathrm{CBH}(2,10,16,34)$. However, to the best of our knowledge, this is the first large sample study taking size of $\mathrm{CBH}$ into account. We demonstrated a higher risk of abnormal composite outcome with increasing size of $\mathrm{CBH}$, infants with massive $\mathrm{CBH}$ having the highest chance of an unfavorable outcome. Even without severe supratentorial injury they still had a very high risk of an abnormal composite outcome. This is in accordance with the results of Limperopoulos et al. (2) and with data shown in the review of Hortensius et al. (12) who both demonstrated that cognitive, language and behavior sequelae occur frequently in infants with isolated $\mathrm{CBH}$. However, in the systematic review of Hortensius et al. (12) an incidence of 43 to $75 \%$ for severe neurodevelopmental outcome in infants with isolated CBH was reported, which is much higher than the $38 \%$ found in this study. This may be explained by the number of infants with punctate $\mathrm{CBH}$ included in the analyses. In the systematic review 15/126 infants with isolated punctate CBH were included, while in our study 60/177 infants were included.

While the composite outcome did not differ significantly between infants with punctate and limited CBH, we found a significant difference in composite outcome between limited and massive CBH. These are important findings. So far, the outcome of infants with limited $\mathrm{CBH}$ has not been reported and previous studies, reporting on the neurodevelopmental outcome of infants with $\mathrm{CBH}$, did not distinguish between limited and massive $\mathrm{CBH}$. Combining these two patterns of $\mathrm{CBH}$ may have influenced the results in previous studies and may erroneously have suggested that limited $\mathrm{CBH}$ carries a similar high risk of an unfavorable prognosis as massive $\mathrm{CBH}$. While 
we found an abnormal composite outcome in $40 \%$ of the infants with limited $\mathrm{CBH}$, this percentage was lower than in infants with massive $\mathrm{CBH}(67 \%)$.

Severe cerebellar atrophy, using the corrected TCD, was seen in $75 \%$ of infants with massive CBH and in a minority of infants with limited or punctate $\mathrm{CBH}$. Due to major growth and development during the second half of gestation, the cerebellum is particularly vulnerable to developmental disruption (4). Also without evident cerebellar injury, mild atrophy may develop in preterm born children. Cerebellar development may be disrupted by various factors i.e. hemorrhage, toxic effects of hemosiderin deposition or supratentorial injury $(5,6,35)$. Not all infants with $\mathrm{CBH}$ developed cerebellar atrophy. It may be interesting and clinically relevant to investigate the pathogenesis of cerebellar atrophy in infants with $\mathrm{CBH}$ and the possible influence of timing of the $\mathrm{CBH}$ in a next, prospective study.

The location of CBH may be of clinical importance in infants with limited or massive CBH. Two studies described a negative effect of vermis involvement in global developmental outcome $(2,9)$, but did not distinguish between limited and massive $\mathrm{CBH}$. While in our study all infants with massive $\mathrm{CBH}$ and vermis involvement had an abnormal composite score, in none of the infants with limited $\mathrm{CBH}$ the vermis was involved. In contrast to Hortensius et al (12), we found a difference in composite outcome between infants with uni- or bilateral CBH, with a more favorable outcome in those with a unilateral $\mathrm{CBH}$. In that study and ours the number of infants with bilateral $\mathrm{CBH}$ was small, therefore no conclusions can be drawn on the relation between laterality and outcome. In infants with punctate $\mathrm{CBH}$, neither the number of lesions nor the location (uni- or bilateral or vermis involvement) had an influence on the composite score.

Several factors have been associated with $\mathrm{CBH}$, such as low GA, low BW, HFO ventilation, inotropic support and severe IVH $(5,6,17,34)$. We tried to identify factors that contributed to limited or massive CBH, when compared to punctate $\mathrm{CBH}$. Mechanical ventilation was independently associated with limited CBH, while lower GA, lower Apgar score and severe thrombocytopenia were independently associated with massive CBH. Comparing limited and massive $\mathrm{CBH}$, most factors were not significantly different between the two groups, suggesting that these may have a more similar pathogenesis. 
We acknowledge several limitations of our study. Firstly, we compared infants with different patterns of CBH,

but we did not compare them with a control group without $\mathrm{CBH}$. Secondly, we retrospectively collected the data of infants with known CBH on MRI. Therefore, this is a selected group of preterm neonates: except for the infants in Utrecht and Milan, MRI was performed when clinically indicated. Infants without or with only minor infra- and supratentorial lesions are therefore underrepresented. However, we have reached our primary aim to compare infants with small, limited and massive CBH.

Thirdly, the participating centers used different scan protocols. At Erasmus MC infants were scanned around PMA 30 weeks, while at the other centers infants were scanned around TEA. This may have influenced the detection rate of small lesions: punctate white matter lesions often fade over time (36), the same may be true for punctate cerebellar lesions. However, we do not think this has influenced our results since in the majority of infants (68\%) SWI was performed, enabling the detection of even tiny (remnants of bleeds after a long period of time and we were still able to compare the different patterns of $\mathrm{CBH}$. Furthermore, we examined cerebellar atrophy around TEA, while this may still develop after this age. Additionally, we only measured TCD and did not measure cerebellar volume and may thus have missed small alterations in cerebellar volumes.

Finally, different neurodevelopmental tests were used. In Italy this was GMDS, while the Dutch centers used BSID-III. Although Picciolini et al. (37) reported that the BSID-III had a higher agreement with GMDS than the BSID-II, there are still differences between the two tests. Italian infants were significantly younger and suffered more neonatal morbidity than Dutch infants, this may partially explain the less favorable outcome in the Italian infants. Moreover, the maternal education level could not be found for more than half of the included infants. This may have influenced test results. In the Dutch centers the language scale of the BSID was not yet validated and could therefore not be used. It is however well known that language may be affected by cerebellar injury (2). This may be another explanation for the less favorable outcome of the Italian infants. All components of the composite outcome were allocated the same weight, but one could argue that for instance CP may impose a larger burden on the infant and its family than an abnormal CBCL score. Although we collected a large population of infants, distributed over 6 centers, the number of infants was still too small to investigate the relation between the specific location of $\mathrm{CBH}$ and neurodevelopmental outcome.

\section{Conclusion}

Limited and massive $\mathrm{CBH}$ are associated with the same perinatal factors. The risk of an abnormal composite outcome increases with increasing size of $\mathrm{CBH}$. Infants with limited $\mathrm{CBH}$ have a more favorable outcome than 
infants with massive $\mathrm{CBH}$. It is therefore important to distinguish between limited and massive $\mathrm{CBH}$. Future studies should focus on the relation between location of $\mathrm{CBH}$ and neurodevelopmental outcome, and between size of $\mathrm{CBH}$ and neurodevelopmental outcome at school age. In addition, the influence of perinatal factors and timing of $\mathrm{CBH}$ on subsequent cerebellar atrophy should be evaluated. 


\section{Acknowledgements}

We are grateful to I.C. van Haastert, O. Picciolini, P. Schiavolin, S. E. Sforza and S. Uccella for their helpful contributions to this project. 


\section{References}

(1) Steggerda SJ, Leijser LM, Wiggers-de Bruïne FT, van dG, Walther FJ, van Wezel-Meijler G. Cerebellar Injury in Preterm Infants: Incidence and Findings on US and MR Images. Radiology 2009;252(1):190-199.

(2) Limperopoulos C, Bassan H, Gauvreau K, Robertson RL,J, Sullivan NR, Benson CB, et al. Does cerebellar injury in premature infants contribute to the high prevalence of long-term cognitive, learning, and behavioral disability in survivors? Pediatrics 2007 Sep;120(3):584-593.

(3) Kidokoro H. Brain injury and altered brain growth in preterm infants: predictors and prognosis. Pediatrics 2014;134(2):444-53.

(4) Volpe JJ. Volpe's neurology of the newborn. Sixth edition. ed. Philadelphia, PA: Elsevier; 2018.

(5) Limperopoulos C, Soul J, Haidar H, Huppi P, Bassan H, Warfield S, et al. Impaired trophic interactions between the cerebellum and the cerebrum among preterm infants. Pediatrics 2005;116(4):844-850.

(6) Volpe JJ. Cerebellum of the premature infant: rapidly developing, vulnerable, clinically important. J Child Neurol 2009;24(9):1085-104.

(7) Tam EWY. Cerebellar injury in preterm infants. Handb Clin Neurol 2018;155:49-59.

(8) Schmahmann JD. Disorders of the cerebellum: ataxia, dysmetria of thought, and the cerebellar cognitive affective syndrome. J Neuropsychiatry Clin Neurosci 2004;16(3):367-78.

(9) Zayek MM, Benjamin JT, Maertens P, Trimm RF, Lal CV, Eyal FG. Cerebellar hemorrhage: a major morbidity in extremely preterm infants. Journal of Perinatology 2012;32(9):699-704.

(10) Bednarek N, Akhavi A, Pietrement C, Mesmin F, Loron G, Morville P. Outcome of cerebellar injury in very low birth-weight infants: 6 case reports. J Child Neurol 2008;23(8):906-911.

(11) Brossard-Racine M, du Plessis AJ, Limperopoulos C. Developmental cerebellar cognitive affective syndrome in ex-preterm survivors following cerebellar injury. Cerebellum, The 2015;14(2):151-64. 
(12) Hortensius L, Dijkshoorn ABC, Ecury Goossen G, Steggerda S, Hoebeek F, Benders, Manon J N L, et al. Neurodevelopmental Consequences of Preterm Isolated Cerebellar Hemorrhage: A Systematic Review. Pediatrics 2018;142(5).

(13) Parodi A. Accuracy of ultrasound in assessing cerebellar haemorrhages in very low birthweight babies. Fetal and Neonatal 2015;100(4):289-92.

(14) Steggerda SJ, van Wezel-Meijler G. Cranial ultrasonography of the immature cerebellum: Role and limitations. Seminars in Fetal and Neonatal Medicine 2016.

(15) Limperopoulos C, Benson CB, Bassan H, Disalvo DN, Kinnamon DD, Moore M, et al. Cerebellar hemorrhage in the preterm infant: ultrasonographic findings and risk factors. Pediatrics 2005 Sep;116(3):717724.

(16) Tam EWY. Cerebellar hemorrhage on magnetic resonance imaging in preterm newborns associated with abnormal neurologic outcome. J Pediatr 2011;158(2):245-50.

(17) Steggerda SJ, De Bruine FT, van dB, Rijken M, Leijser LM, Walther FJ, et al. Small cerebellar hemorrhage in preterm infants: perinatal and postnatal factors and outcome. Cerebellum 2013 Dec;12(6):794-801.

(18) Meijler G, Steggerda SJ. Neonatal Cranial Ultrasonography. 3rd ed. ed. Cham: Springer; 2019.

(19) Schmahmann JD, Doyon J, McDonald D, Holmes C, Lavoie K, Hurwitz AS, et al. Three-dimensional MRI atlas of the human cerebellum in proportional stereotaxic space. Neuroimage 1999;10(3):233-60.

(20) Koziol LF, Budding D, Andreasen N, D'Arrigo S, Bulgheroni S, Imamizu H, et al. Consensus paper: the cerebellum's role in movement and cognition. Cerebellum, The 2014;13(1):151-77.

(21) Stoodley CJ, Limperopoulos C. Structure-function relationships in the developing cerebellum: Evidence from early-life cerebellar injury and neurodevelopmental disorders. Seminars in Fetal and Neonatal Medicine 2016.

(22) Klein AP, Ulmer JL, Quinet SA, Mathews V, Mark LP. Nonmotor Functions of the Cerebellum: An Introduction. Am J Neuroradiol 2016;37(6):1005-9. 
(23) Hoftiezer L, Hukkelhoven, Chantal W P M, Hogeveen M, Straatman, Huub M P M, van Lingen R. Defining small-for-gestational-age: prescriptive versus descriptive birthweight standards. Eur J Pediatr 2016;175(8):10471057.

(24) Martin RR. Massive intracerebellar hemorrhage in low-birth-weight infants. Journal of Pediatrics, The $1976 ; 89(2): 290-3$

(25) Kidokoro H. New MR imaging assessment tool to define brain abnormalities in very preterm infants at term. Am J Neuroradiol 2013;34(11):2208-14.

(26) Bolduc M, du Plessis A, Sullivan N, Guizard N, Zhang X, Robertson R, et al. Regional cerebellar volumes predict functional outcome in children with cerebellar malformations. Cerebellum 2012;11(2):531-542.

(27) Volpe JJ. Intraventricular hemorrhage in the premature infant--current concepts. Part II. Ann Neurol 1989;25(2):109-16.

(28) Bayley N. Bayley Scales of Infant and Toddler Development. . 3rd edition ed. San Antonio, TX: Harcourt Assessment; 2006.

(29) Achenbach TM, Rescorla L. Manual for the Child Behavior Checklist. Preschool Forms and Profiles. : Burlington VT: University of Vermont Department of Psychiatry; 2000.

(30) Griffiths R, Huntley M. \&nbsp;The Griffiths mental development scales-revised manual: from birth to 2 years. High Wycombe: Association for Research in Infant and Child Development; 1996.

(31) Palisano R, Rosenbaum P, Walter S, Russell D, Wood E, Galuppi B. Development and reliability of a system to classify gross motor function in children with cerebral palsy. Dev Med Child Neurol 1997;39(4):21423.

(32) Dekker MC. Emotional and behavioral problems in children and adolescents with and without intellectual disability. Journal of Child Psychology and Psychiatry 2002;43(8):1087-98.

(33) van dB, Kok JH, Houtzager BA, Scherjon SA. Behavioural problems at the age of eleven years in pretermborn children with or without fetal brain sparing: a prospective cohort study. Early Hum Dev 2010;86(6):379-84. 
(34) Dyet L, Kennea N, Counsell S, Maalouf E, Ajayi Obe M, Duggan P, et al. Natural history of brain lesions in extremely preterm infants studied with serial magnetic resonance imaging from birth and neurodevelopmental assessment. Pediatrics 2006;118(2):536-548.

(35) Limperopoulos C, Soul JS, Gauvreau K, Huppi PS, Warfield SK, Bassan H, et al. Late Gestation Cerebellar Growth Is Rapid and Impeded by Premature Birth. Pediatrics 2005;115(3):688-695.

(36) Kersbergen K, Benders, Manon J N L, Groenendaal F, Koopman Esseboom C, Nievelstein RAJ, van Haastert I, et al. Different patterns of punctate white matter lesions in serially scanned preterm infants. PLoS ONE 2014;9(10):e108904.

(37) Picciolini O, Squarza C, Fontana C, Giannì M, Cortinovis I, Gangi S, et al. Neurodevelopmental outcome of extremely low birth weight infants at 24 months corrected age: a comparison between Griffiths and Bayley Scales. BMC Pediatr 2015;15:139. 


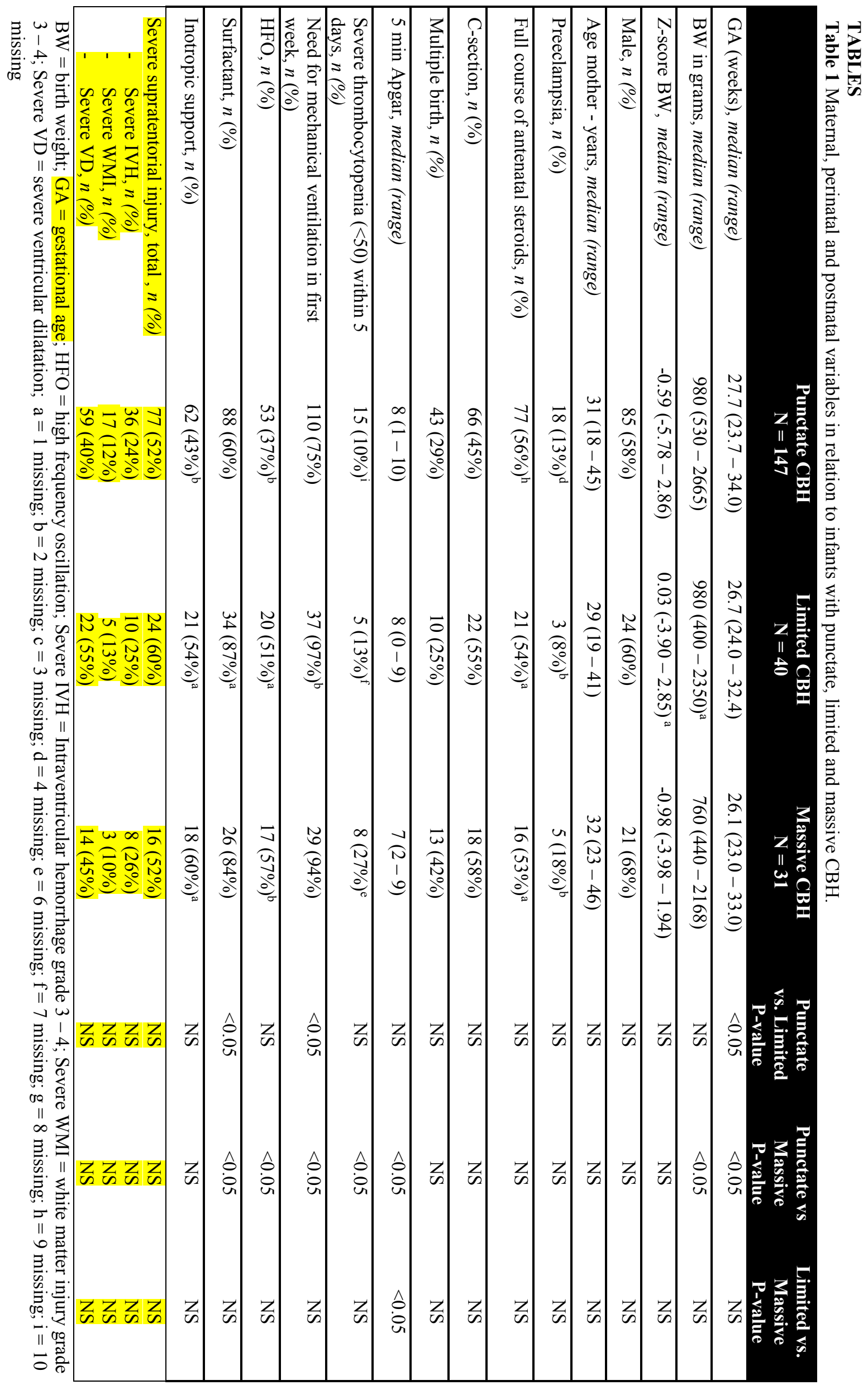


Table 2 Mean transcerebellar diameter (TCD) around TEA (38 - 44 weeks) per pattern of CBH.

\begin{tabular}{|l|l|}
\hline CBH pattern & TCD $(\mathbf{m m})$, median (range) \\
\hline Punctate $(\mathrm{N}=102)$ & $51.9(40.9-58.1)$ \\
\hline Limited $(\mathrm{N}=23)$ & $46.5(40.9-56.8)$ \\
\hline Massive $(\mathrm{N}=16)$ & $38.1(22.3-57.5)$ \\
\hline
\end{tabular}

Table 3 Follow- up: number of cases per type of test.

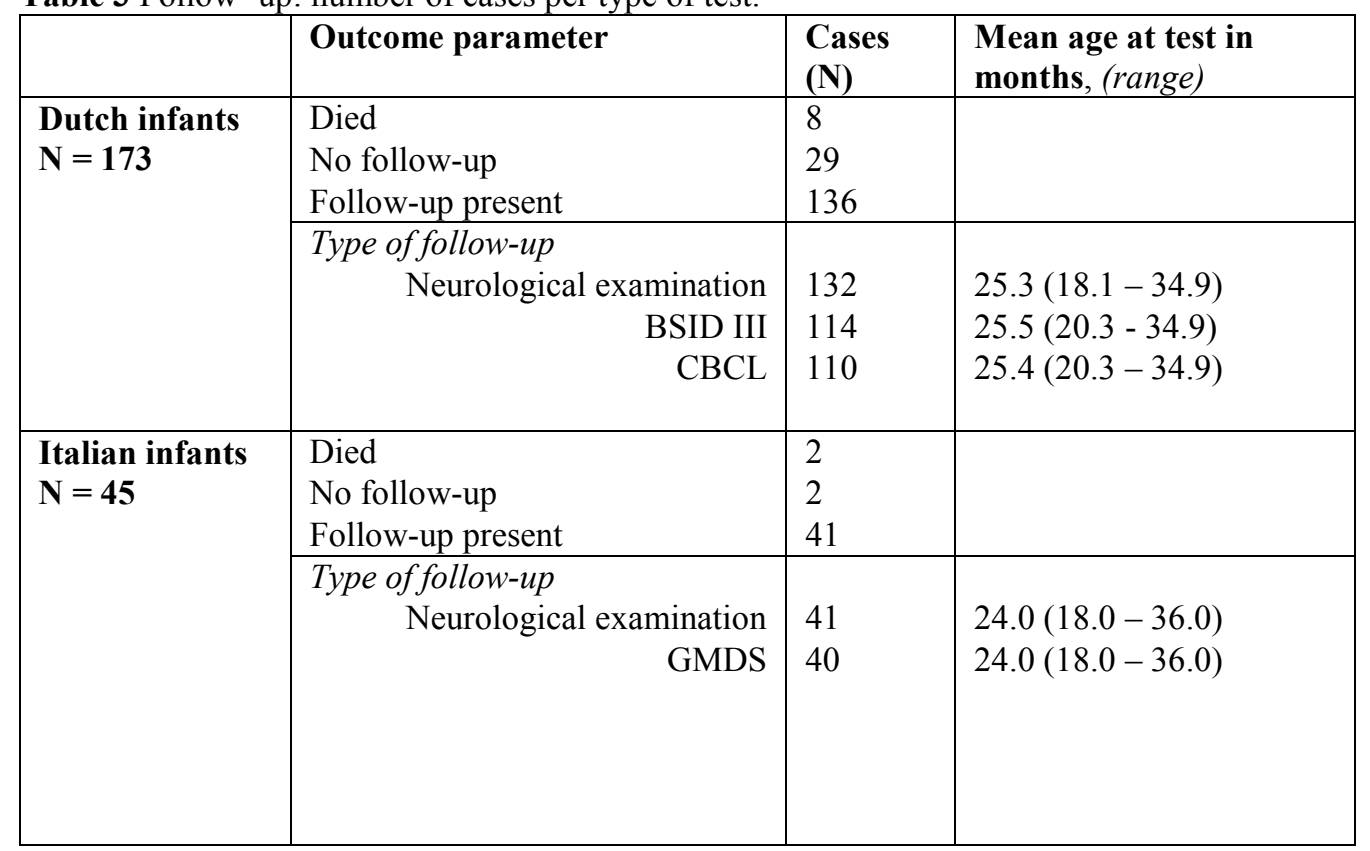

Table 4 Abnormal test results of infants with punctate, limited and massive $\mathrm{CBH}$ and infants.

\begin{tabular}{|c|c|c|c|c|c|c|c|}
\hline \multirow[b]{2}{*}{ Type of test } & \multicolumn{2}{|c|}{ Punctate CBH } & \multicolumn{2}{|c|}{ Limited CBH } & \multicolumn{2}{|c|}{ Massive CBH } & \multirow[t]{2}{*}{ P-value* } \\
\hline & $\begin{array}{l}\text { Total infants } \\
\text { tested, } \mathrm{N}\end{array}$ & $\begin{array}{l}\text { \% abnormal } \\
\text { test result }\end{array}$ & $\begin{array}{c}\text { Total infants } \\
\text { tested, } \mathrm{N}\end{array}$ & $\begin{array}{l}\% \text { abnormal } \\
\text { test result }\end{array}$ & $\begin{array}{l}\text { Total infants } \\
\text { tested, N }\end{array}$ & $\begin{array}{l}\text { \% abnormal } \\
\text { test result }\end{array}$ & \\
\hline $\begin{array}{l}\text { Neurological } \\
\text { examination }\end{array}$ & 116 & $15 \%$ & 35 & $26 \%$ & 22 & $36 \%$ & 0.04 \\
\hline BSID-III & 91 & $21 \%$ & 15 & $20 \%$ & 8 & $50 \%$ & 0.16 \\
\hline GMDS & 13 & $54 \%$ & 16 & $69 \%$ & 11 & $91 \%$ & 0.14 \\
\hline CBCL & 88 & $17 \%$ & 14 & $21 \%$ & 8 & $25 \%$ & 0.81 \\
\hline $\begin{array}{l}\text { Composite } \\
\text { score** }\end{array}$ & 119 & $40 \%$ & 35 & $51 \%$ & 23 & $78 \%$ & $<0.01$ \\
\hline
\end{tabular}

$* \mathrm{X}^{2}$-test.

** If at least one of the separate tests was abnormal. 
Table 5 Maternal, perinatal and postnatal variables of Dutch and Italian infants

\begin{tabular}{|c|c|c|c|}
\hline & $\begin{array}{l}\text { Dutch infants } \\
N=173\end{array}$ & $\begin{array}{l}\text { Italian infants } \\
\mathrm{N}=45\end{array}$ & P-value \\
\hline GA (weeks), median (range) & $27.4(24.0-34.0)$ & $26.5(23.0-32.4)$ & 0.02 \\
\hline $\mathrm{BW}(\mathrm{g})$, median (range) & $1000(400-2665)$ & $1087(440-2350)^{\mathrm{a}}$ & 0.03 \\
\hline Z-score for BW, median (range) & $-0.48(-5.78-2.86)$ & $-0.78(-3.75-2.85)^{\mathrm{a}}$ & 0.62 \\
\hline Male, $n(\%)$ & $101(58 \%)$ & $29(64 \%)$ & 0.46 \\
\hline Age mother - years, median (range) & $31(18-41)$ & $32(29-46)$ & 0.01 \\
\hline Preeclampsia, $n(\%)$ & $23(14 \%)^{\mathrm{c}}$ & $3(8 \%)^{\mathrm{e}}$ & 0.32 \\
\hline Full course of antenatal steroids $n(\%)$ & $93 \quad(56 \%)^{\mathrm{f}}$ & $21(51 \%)^{d}$ & 0.58 \\
\hline C-section, $n(\%)$ & $74(43 \%)$ & $32(71 \%)$ & $<0.01$ \\
\hline Multiple birth, $n(\%)$ & $48(28 \%)$ & $18(40 \%)$ & 0.11 \\
\hline 5 min Apgar, median (range) & $8(1-10)$ & $8(0-10)$ & 0.77 \\
\hline $\begin{array}{l}\text { Severe thrombocytopenia }(<50) \text { within } 5 \\
\text { days, } n(\%)\end{array}$ & $25(16 \%)^{j}$ & $3(8 \%)^{g}$ & 0.23 \\
\hline $\begin{array}{l}\text { Need for mechanical ventilation in first week, } \\
n(\%)\end{array}$ & $139(80 \%)$ & $37(86 \%)$ & 0.39 \\
\hline $\mathrm{HFO}, n(\%)$ & $67(39 \%)^{\mathrm{a}}$ & $23(56 \%)^{d}$ & 0.05 \\
\hline Surfactant, $n(\%)$ & $116(67 \%)$ & $32(73 \%)^{\mathrm{a}}$ & 0.47 \\
\hline Postnatal steroids, $n(\%)$ & $44(27 \%)^{\mathrm{a}}$ & $23(55 \%)^{\mathrm{c}}$ & $<0.001$ \\
\hline Inotropic support, $n(\%)$ & $78(45 \%)^{\mathrm{a}}$ & $23(55 \%)^{\mathrm{c}}$ & 0.27 \\
\hline $\begin{array}{l}\text { Severe Supratentorial Injury } \\
\text { Total }\end{array}$ & $97 \quad(56 \%)$ & $20(44 \%)$ & 0.16 \\
\hline Punctate, $n(\%)$ & $71 \quad(54 \%)$ & $6 \quad(40 \%)$ & 0.88 \\
\hline Limited, $n(\%)$ & $16 \quad(73 \%)$ & $8 \quad(44 \%)$ & 0.07 \\
\hline Massive, $n(\%)$ & $10 \quad(53 \%)$ & $6 \quad(50 \%)$ & 0.31 \\
\hline Surgery before TEA, $n(\%)$ & $45 \quad(26 \%)$ & $19(42 \%)$ & 0.03 \\
\hline
\end{tabular}

$\mathrm{BW}=$ birth weight; GA = gestational age; $\mathrm{HFO}=$ high frequency oscillation; TEA $=$ term equivalent age; $\mathrm{a}=1$ missing; $b=2$ missing; $c=3$ missing; $d=4$ missing; $e=6$ missing; $f=7$ missing; $g=8$ missing; $h=9$ missing; $i$ $=10$ missing $\mathrm{j}=15$ missing 
Table 6 Abnormal test results of infants with punctate, limited and massive CBH without severe supratentorial injury.

\begin{tabular}{|c|c|c|c|c|c|c|c|}
\hline \multirow[b]{2}{*}{ Type of test } & \multicolumn{2}{|c|}{ Punctate CBH } & \multicolumn{2}{|c|}{ Limited CBH } & \multicolumn{2}{|c|}{ Massive CBH } & \multirow[t]{2}{*}{ P-value* } \\
\hline & $\begin{array}{l}\text { Total infants } \\
\text { tested, } \mathrm{N}\end{array}$ & $\begin{array}{l}\text { \% abnormal } \\
\text { test result }\end{array}$ & $\begin{array}{c}\text { Total infants } \\
\text { tested, } \mathrm{N}\end{array}$ & $\begin{array}{l}\% \text { abnormal } \\
\text { test result }\end{array}$ & $\begin{array}{l}\text { Total infants } \\
\text { tested, } \mathrm{N}\end{array}$ & $\begin{array}{l}\text { \% abnormal } \\
\text { test result }\end{array}$ & \\
\hline $\begin{array}{l}\text { Neurological } \\
\text { examination }\end{array}$ & 59 & $5 \%$ & 15 & $13 \%$ & 11 & $18 \%$ & 0.14 \\
\hline BSID-III & 49 & $10 \%$ & 4 & $0 \%$ & 4 & $50 \%$ & 0.14 \\
\hline GMDS & 7 & $71 \%$ & 9 & $55 \%$ & 6 & $83 \%$ & 0.63 \\
\hline CBCL & 45 & $11 \%$ & 4 & $25 \%$ & 4 & $25 \%$ & 0.28 \\
\hline $\begin{array}{l}\text { Composite } \\
\text { score** }\end{array}$ & 60 & $25 \%$ & 15 & $40 \%$ & 12 & $67 \%$ & 0.02 \\
\hline
\end{tabular}

$* *$ If at least one of the separate tests was abnormal.

Table 7 Abnormal composite test results of infants with unilateral or bilateral CBH.

\begin{tabular}{|l|c|c|c|c|c|c|}
\hline \multicolumn{2}{|l|}{ Punctate CBH } & \multicolumn{2}{l|}{ Limited CBH } & \multicolumn{2}{l|}{ Massive CBH } \\
\hline $\begin{array}{l}\text { Composite } \\
\text { score }\end{array}$ & Unilateral & Bilateral & Unilateral & Bilateral & Unilateral & Bilateral \\
\hline Normal & $40(62 \%)$ & $30(64 \%)$ & $15(52 \%)$ & $2(33 \%)$ & $5(26 \%)$ & $0(0 \%)$ \\
Abnormal & $24(38 \%)$ & $17(36 \%)$ & $14(48 \%)$ & $4(67 \%)$ & $14(74 \%)$ & $4(100 \%)$ \\
\hline
\end{tabular}

Table 8 Abnormal composite test results of infants with or without vermis involvement

\begin{tabular}{|l|c|l|l|l|l|l|}
\hline \multicolumn{2}{|l|}{ Punctate CBH } & \multicolumn{2}{l|}{ Limited CBH } & \multicolumn{2}{l|}{ Massive CBH } \\
\hline $\begin{array}{l}\text { Composite } \\
\text { score }\end{array}$ & $\begin{array}{l}\text { No vermis } \\
\text { involvement }\end{array}$ & $\begin{array}{l}\text { Vermis } \\
\text { involvement }\end{array}$ & $\begin{array}{l}\text { No vermis } \\
\text { involvement }\end{array}$ & $\begin{array}{l}\text { Vermis } \\
\text { involvement }\end{array}$ & $\begin{array}{l}\text { No vermis } \\
\text { involvement }\end{array}$ & $\begin{array}{l}\text { Vermis } \\
\text { involvement }\end{array}$ \\
\hline Normal & $60(61 \%)$ & $12(60 \%)$ & $17(49 \%)$ & $0(0 \%)$ & $5(33 \%)$ & $0(0 \%)$ \\
Abnormal & $39(39 \%)$ & $8(40 \%)$ & $18(51 \%)$ & $0(0 \%)$ & $10(67 \%)$ & $8(100 \%)$ \\
\hline
\end{tabular}




\section{TEXT FIGURES}

Figure 1. MR images performed around TEA. A) T2-weighted MR image and SWI of a punctate CBH located in the right cerebellar hemisphere (Arrow). B) Limited $\mathrm{CBH}$ at the convexity of the left cerebellar hemisphere. C) Massive $\mathrm{CBH}$ located in the left cerebellar hemisphere, also leading to destruction and atrophy of that hemisphere.

Figure 2. Map of structural-function corresponding regions on transverse (1) and coronal (2) planes. A. Convexity (lateral-posterior-inferior) hemispheric zones; B. Anterior/medial hemispheric zones; C. Vermis. 


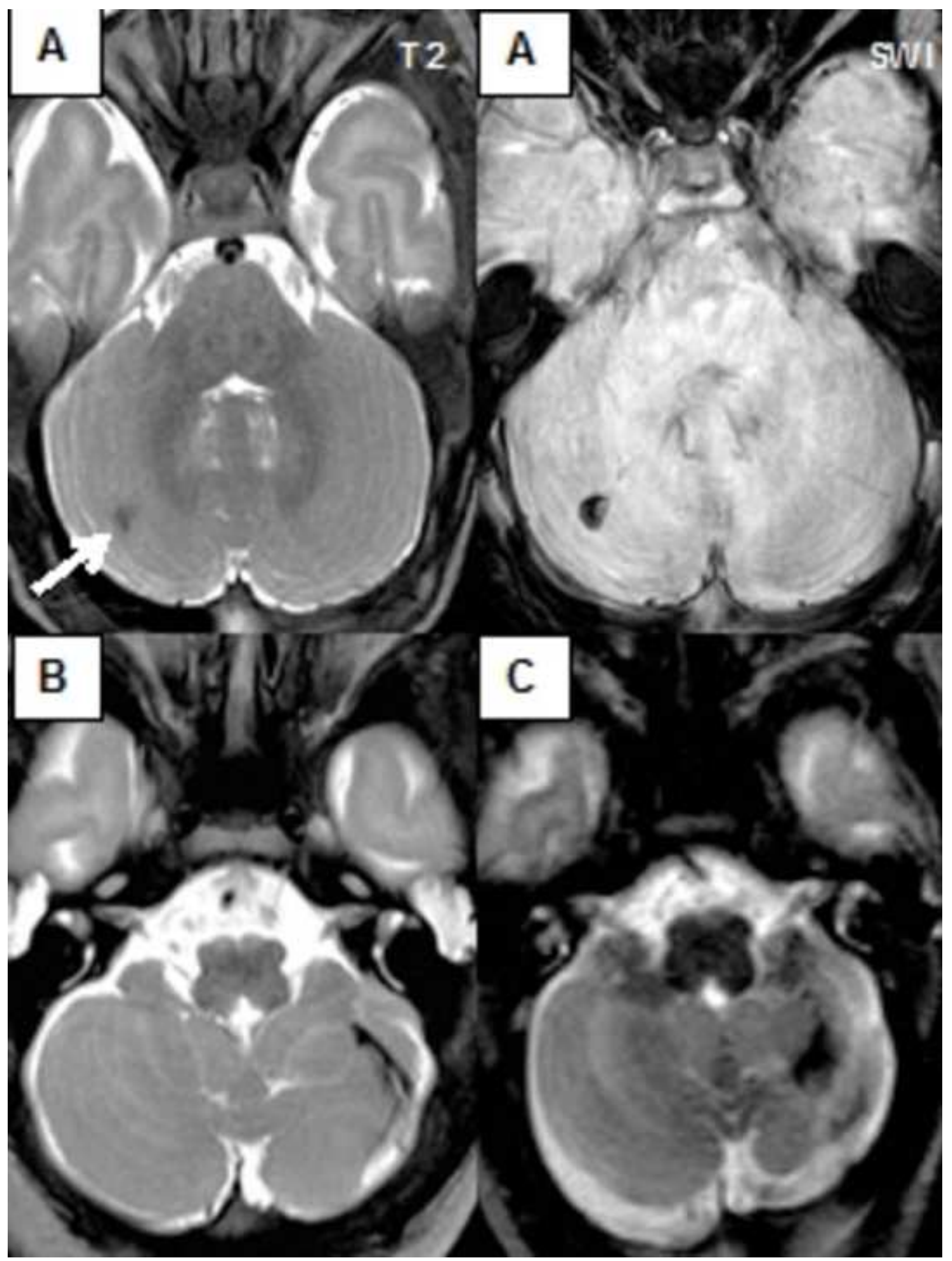




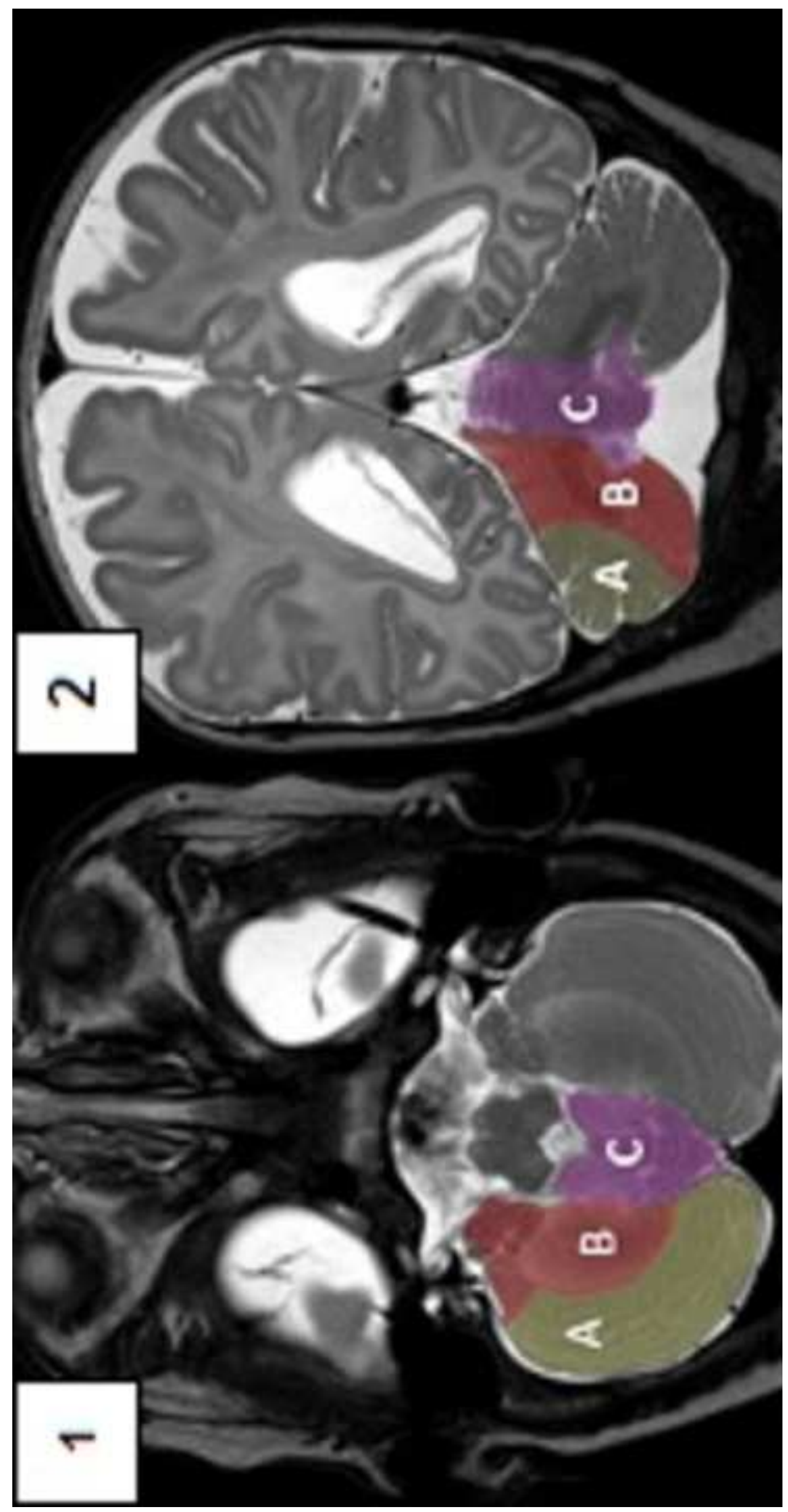

